\section{PTH-102 FERRIC MALTOL, UNLIKE FERROUS SULPHATE, DOES NOT ADVERSELY AFFECT THE INTESTINAL MICROBIOME}

${ }^{1}$ Awad Mahalhal ${ }^{*},{ }^{2}$ John Mansfield, ${ }^{3}$ Mark Sampson, ${ }^{4}$ Stephen Lewis, ${ }^{2}$ Chris Lamb, ${ }^{1}$ Alessandra Frau, ${ }^{1} \mathrm{DM}$ Pritchard, ${ }^{1}$ Chris Probert. ${ }^{1}$ Gastroenterology Research Unit, Institute of Translational Medicine, University of Liverpool, Liverpool, UK; ${ }^{2}$ Gastroenterology, Newcastle upon Tyne Hospitals NHS Foundation Trust, Newcastle upon Tyne, UK; ${ }^{3}$ Shield Therapeutics, London, UK; ${ }^{4}$ Nuffield Health Plymouth Hospital, Plymouth, UK

\subsection{6/gutjnl-2019-BSGAbstracts. 161}

Introduction We have previously shown that altering dietary ferrous iron consumption exacerbates murine models of inflammatory bowel disease (Mahalhal et al., 2018) and is associated with dysbiosis. We have now investigated the effect of oral ferric maltol and ferrous sulphate on the microbiome of patients with iron deficiency anaemia (IDA) and on mice treated with dextran sodium sulphate (DSS) to induce colitis. We report results of changes at the phylum level.

Methods Studies were performed on three groups of wild-type mice: acute colitis was induced with $2 \%$ DSS for 5 days, followed by 5 further days on water while mice were fed one of three diets (from day-1 of DSS treatment): normal diet (200ppm iron ferrous sulphate) [ND $(n=8)]$, 400ppm iron ferrous sulphate [FS $(n=16)]$ or $400 \mathrm{ppm}$ iron ferric maltol [FM $(n=16)]$. Clinical and pathological data were compared at day1 and day-10, when faecal samples were collected. 16 patients with IDA were also recruited: 10 were treated with FS and 6 with FM supplements for 4 weeks: paired faecal samples (pre and post-treatment) were collected. Faecal bacterial gDNA was extracted from murine and human samples and the microbiota composition was determined from the sequence of V4 region of $16 \mathrm{~S}$ rDNA. Statistical inferences were made using Welch's t-test with post-hoc analysis: Shannon Diversity Index (SDI) and Principal Component Analysis (PCA) were used to compare population and phylum-level changes.

Results DSS-induced colitis was worse in ND and FS mice than FM mice (determined by weight loss [3, $7 \& 0 \%$, at day-8 respectively] and histology [median score $2,2 \& 1$ at day-10]). FS supplementation was associated with an increase in Bacteroidetes (15\% in mice, $4 \%$ in humans) whereas FM led to a reduction in Bacteroidetes (3\% in mice and $15 \%$ in humans). There was a $4 \%$ increase in Firmicutes with FM supplementation in mice and $20 \%$ in humans whereas FS led to a $15 \%$ reduction in Firmicutes in mice and 5\% in humans. Conclusion This study has demonstrated differential and unique influences of ferric maltol and ferrous sulphate supplements on the faecal microbiome of mice with DSS induced colitis and patients with iron deficiency. These differences might contribute to the difference in side effects that are associated with these preparations.

\section{PTH-103 THE GASTROENTEROLOGIST'S BIGGEST FEAR - WHEN THE CURE IS WORSE THAN THE DISEASE}

Aarani Mahalingam, Sophie Smith*, Ioannis Koumoutsos, Jonas Woo. Southend Hospital, Southend, UK

\subsection{6/gutjnl-2019-BSGAbstracts. 162}

Introduction One of the most sinister, whilst rare, complications of combination treatment with biological and thiopurines therapy is lymphoproliferative disorders. We present a case of a 35 year old gentleman with a background of Crohn's disease. He presented with symptoms of chest pain, dyspnoea and an atypical rash, to the gastroenterology ward. Following extensive investigations he was diagnosed with hepatosplenic T-cell lymphoma.

The patient had a longstanding history of penetrating small bowel Crohn's disease with two previous small bowel resections and multiple stricturoplasties. After failing monotherapy with Azathioprine, he was started on combination treatment with Adalimumab and Azathioprine, which brought his disease into symptomatic remission for 5 years.

On presentation to $\mathrm{A} \& \mathrm{E}$, in addition to the symptoms detailed above, he had a 3 month history of $\mathrm{B}$ symptoms (fevers, night sweats and weight loss). Therefore, on consideration of diagnosis our top differential was of possible haematological malignancy whilst his cutaneous manifestation led us to consider inflammatory or vasculitic processes.

Results Radiologic investigations including computed tomography scan [CT] and positron emission tomography scan [PET] showed long standing hepatosplenomegaly with portal hypertension, but with no evidence of lymphadenopathy, malignancy or large vessel vasculitis. He continued to spike fevers and showed evidence of haemolysis and severe anaemia requiring daily transfusions. His lactate dehydrogenase [LDH] was over $9600 \mathrm{U} / \mathrm{L}$ initially and then continued to rise above $15000 \mathrm{U} / \mathrm{L}$, and his ferritin was significantly elevated at $12025 \mathrm{ng} / \mathrm{ml}$ with otherwise minimally raised inflammatory markers.

The main differentials remained haematological including lymphoma or Hemophagocytic Lymphohistiocytosis. He subsequently had a bone marrow biopsy which confirmed the diagnosis of hepatosplenic T-Cell lymphoma.

$\mathrm{He}$ was transferred to a tertiary centre where he was treated with chemotherapy and was deemed to be in remission, awaiting sibling allograft.

Conclusion Literature suggests the risk of Hepatosplenic T-cell lymphomas [HSTCLs] occur almost exclusively in males under the age of 35 who are exposed to thiopurines and the risk increases for those treated with combination of thiopurines and anti-TNF agents. In the latter group risk increases when the duration of combination treatment exceeds two years. Therefore, question should be posed whether we should avoid combination therapy in this cohort of patients for more than two years or whether alternative concomitant immunomodulation or biologics should be considered in high risk cases.

\section{PTH-104 REMISSION AND RECURRENCE IN IBD AFTER BIOLOGICAL THERAPY}

S Mankodi* , P Patel, J Gertner, D Nissan, D Sadigh, CM Onnie. Whittington NHS Trust, London, UK

\subsection{6/gutjnl-2019-BSGAbstracts. 163}

Introduction Biological agents have revolutionised the treatment of inflammatory bowel disease (IBD) with usage continually rising. Decisions regarding de-escalation of therapy require balancing the financial implications, risks of long-term immunosuppression against the risk of relapse, and the potential of unsuccessful re-challenge. Approximately one third of IBD patients relapse within 12 months of discontinuing biologics. ${ }^{1}$ We aimed to identify the factors associated with relapse and remission.

Methods The IBD register at a single district general hospital was analysed for all patient who had received biologic therapy 


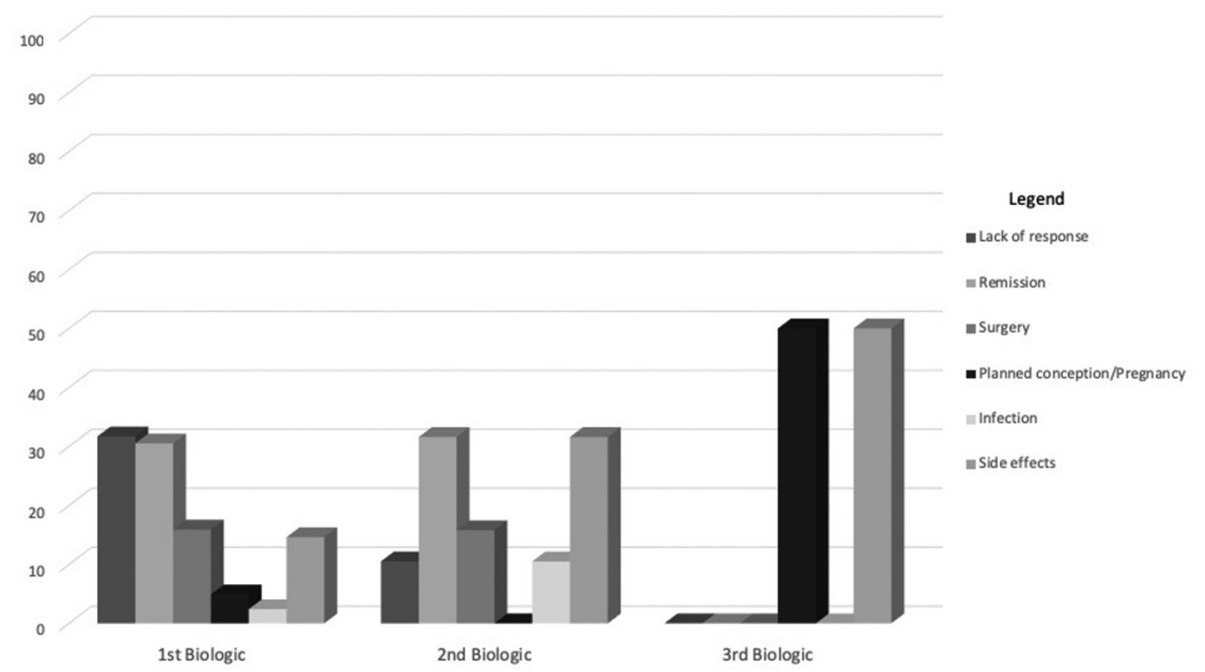

Abstract PTH 104 Figure 1: Rationale for cessation of biological therapy

between 2015-2018. Details of current and previous biologic therapy and concurrent immunosuppression was recorded.

Results 158 patients (69.6\% Crohn's disease (CD) $40.4 \%$ ulcerative colitis (UC)) required biologic treatment (BT) for IBD. $51.8 \%$ of these patients stopped BT over this time period. The median duration of BT was 16 months (IQR 6-35). Of these 60 and 11 patients required a second and third agent respectively. $96.2 \%$ of patients who stopped BT were on an anti-TNF agent $(p=0.001)$. The reasons for changing therapies are detailed in Figure 1. Patients with CD were 2.82 times $(95 \% \mathrm{CI} 1.09-7.33, \mathrm{p}=0.033)$ more likely to lose response to biological therapy than patients with UC. The odds ratio of losing response to $\mathrm{BT}$ in those patients on infliximab compared with alternative agents was 4.11 (95\% CI 0.89-19.01, $\mathrm{p}=0.071)$. Patients on infliximab were 6.39 times (95\%CI $0.80-51.13, \mathrm{p}=0.081)$ more likely to lose response to BT than those on adalimumab. There were no other significant differences between biological agents and other reasons for stopping therapy.

32 cases $(55.1 \%)$ were identified as having discontinued treatment due to clinical remission. Of these, 12 cases were thiopurine naïve previously. 24 cases had been followed up for over 12 months since discontinuation. The within 12 month relapse rate was $37.5 \%$ and the median time to relapse was 9 months (IQR 6-13.5). 93.3\% of patients who relapsed were established on an immunomodulator prior to remission. There was no significant difference in the rate of relapse between step-up vs step-down therapy $(p=0.647)$. Fifteen cases were re-challenged with the same biologic for relapse after successful remission, $80 \%$ of whom had a successful rechallenge.

Conclusions Findings in our centre are comparable to the recognised rate of patients relapsing within a year of achieving remission and discontinuing therapy. There was no significant difference in the rate of relapse in patients who achieved remission between those who were stepped up or stepped down to biologics. The higher proportion of patients who lost response to infliximab may be a consequence of the drug's higher immunogenicity.

\section{REFERENCE}

1. Kennedy, et al. APT 2016;43(8):910-923.

\section{PTH-105 HYPERGLYCAEMIA IN STEROID TREATED HOSPITALISED IBD INPATIENTS AND ITS RISK FACTORS IDENTIFIED BY MACHINE LEARNING}

${ }^{1}$ Martin McDonnell*, 'Richard Harris, ${ }^{1}$ Suranga Dharmasiri, ${ }^{2}$ Hang Phan, ${ }^{2}$ Florina Borca, Tilly Mills, 'Louise Downey, 'David Young, 'Shahid Shahid, 'Robert Livingstone, ${ }^{1}$ Richard Felwick, ${ }^{1,3}$ Fraser Cummings, 'Markus Gwiggner. 'University Hospital Southampton, Southampton, UKi ${ }^{2}$ University of Southampton NIHR Biomedical Research Facility, Southampton, UK; ${ }^{3}$ University of Southampton, Southampton, UK

\subsection{6/gutjnl-2019-BSGAbstracts. 164}

Introduction Glucocorticoids (GC) have been first line treatment for hospitalised IBD patients for over 60 years, despite the introduction of biologic therapy. IBD patients often have systemic inflammation complicated by malnutrition leading to metabolic stress. Frequency and risk factors for hyperglycaemia in hospitalised IBD patients receiving GC are unknown.

Methods 112 consecutive IBD inpatients receiving intravenous hydrocortisone (IVH) for acute flares had capillary blood glucose (CBG) monitoring automatically triggered by the electronic prescription. CBG, biomarkers, IBD severity scores (Harvey Bradshaw, partial Mayo) and weight loss were prospectively recorded. Undiagnosed Diabetes Mellitus (DM) was defined as $\mathrm{HbA} 1 \mathrm{c}>48 \mathrm{mmol} / \mathrm{mol}$. Machine learning (random forest regressor, RFR) was applied to data to evaluate risk factors of hyperglycaemia. 
Results $51 \%$ of hospitalised IVH treated IBD patients met the WHO criteria of DM (CBG>11 mmol/L), while $20 \%$ and $6 \%$ had a CBG $>14 \mathrm{mmol} / \mathrm{l}$ and $>20 \mathrm{mmol} / \mathrm{l}$, respectively. 8 patients had pre-existing DM, which was confirmed by admission $\mathrm{HbA1c}$. RFR indicated disease severity score, duration of IVH, HbA1c and electrolyte imbalances (64\%) were best predictors of hyperglycaemia. $49 \%$ were started on or switched biological therapy during admissions. 55\% were discharged on prednisolone, $14 \%$ on budesonide and $34 \%$ on no GC. 48 patients had HbA1c checked at 3 month follow-up of which 4 were in the diabetic range. 1 was known DM with elevated CBG during admission whose insulin had been titrated, 2 had elevated $\mathrm{CBG}$ as inpatients with no prior DM discharged on gliclazide and insulin respectively and 1 was on long-term steroids for asthma who did not have CBG $>11.0 \mathrm{mmol} / \mathrm{L}$ as inpatient. 4 other patients discharged on gliclazide for steroid induced DM had documented repeat HbA1c recorded, which were all in the normal range.

Conclusions Our data demonstrates that hyperglycaemia is common in IVH treated inpatients, therefore CBG monitoring should be routine practice. Predictive modelling (RFR) identifies more severe disease activity, duration of IVH treatment and $\mathrm{HbA1c}$ as risk factors for hyperglycaemia. The importance of IVH duration suggests hyperglycaemia risk may be physician-modifiable. Alternative treatment strategies such as earlier introduction of biologics, rapid steroid taper and nutritional support could be used to minimise medication associated metabolic instability in high risk patients.

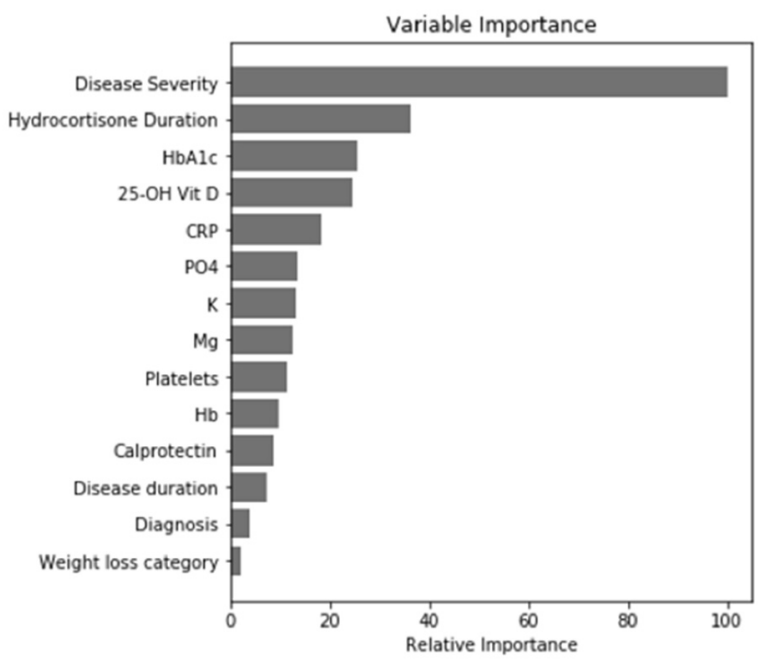

Abstract PTH 105 Figure 1 Relative importance of input features of RFR model for prediction of highest CBG

\section{PTH-106 PREDICTING RESPONSE TO VEDOLIZUMAB IN CROHN'S DISEASE - A SINGLE CENTRE EXPERIENCE}

${ }^{2}$ Claire Kane, ${ }^{1}$ Ross J Porter, ${ }^{2} J o h n$ Thomson, ${ }^{2}$ Malcolm Smith, ${ }^{2}$ Umesh Basavaraju, ${ }^{2}$ Gillian H Bain, ${ }^{1,2}$ Mairi H McLean*. ${ }^{1}$ University Of Aberdeen, Aberdeen, UK; ${ }^{2}$ Aberdeen Royal Infirmary, Aberdeen, UK

\subsection{6/gutjn--2019-BSGAbstracts. 165}

Introduction There is a clinical need to develop personalised clinical biomarker scoring systems to direct biologic treatment for Crohn's disease (CD). Vedolizumab is a humanised anti- $\alpha$ $4 \beta 7$ integrin biologic, inhibiting migration of lymphocytes to the gastrointestinal mucosa. Dulai and colleagues [Gastroenterology, 2018; 155(3)] developed a new clinical prediction tool to determine probability of response to Vedolizumab. Our aim was to retrospectively determine whether this published clinical prediction tool could identify responders to Vedolizumab in our single tertiary IBD centre.

Methods Patients receiving Vedolizumab for $\mathrm{CD}$ between 2015-2018 were identified from the IBD database. The Dulai clinical score $(>19$ predicting response and $\leq 13$ predicting non-response) was retrospectively calculated from baseline pretreatment characteristics including absence of previous antiTNF therapy $(+3)$, no previous gastrointestinal surgery $(+2)$, no fistulising disease $(+2)$ along with pre-treatment CRP (mg/ L) (variable) and albumin levels $(\mathrm{g} / \mathrm{L})$ (variable) as previously published. Clinical response at 26 weeks was determined based on documented improvement in clinical symptoms and no requirement for steroids. Radiological or endoscopic improvement in disease activity and reduction in faecal calprotectin were considered if data was available. Analysis was performed with SPSS V25 with Mann-Whitney U test, Fisher's exact test and ROC analysis, with significance $\mathrm{p}<0.05$.

Results Forty-nine patients with CD received Vedolizumab. 10 were excluded from analysis as they did not reach the 26week endpoint. Following 26 weeks of Vedolizumab treatment, 13/39 (33.3\%) CD patients had evidence of response. Responders versus non-responders had a median pre-treatment predictive score of 16.3 (8.2-20.8) and 14.1 (6.6-13.5), respectively $(\mathrm{p}=0.222)$. ROC analysis reported area under the curve of 0.621 (95\% CI $0.44-0.81)(\mathrm{p}=0.222)$. A score $>19$ had poor sensitivity [23.08\% (95\% CI 50.38,53.81)] but good specificity $[84.62 \% \quad(95 \%$ CI $65.13,95.64)]$ for predicting response to therapy. A score $\leq 13$ had poor sensitivity $[42.31 \%$ (95\% CI 22.35, 63.08)] but good specificity [84.62\% (95\% CI 54.55, 98.08)] for predicting non-response. There was no association between predictive score $>19$ or $\leq 13$ and patients who did $(p=0.666)$ or did not $(p=0.151)$ respond to Vedolizumab therapy, respectfully.

Conclusions In this retrospective single centre tertiary IBD centre analysis, the previously published Dulai clinical score did not predict response to Vedolizumab in CD. Future prospective analysis and consideration of a modified score including additional parameters is warranted.

\section{PTH-107 A UK NATIONAL SURVEY OF THERAPEUTIC DRUG MONITORING WITH ANTI-TNF MEDICATIONS IN IBD}

${ }^{1}$ Shadab Nayeemuddin*, ${ }^{1}$ Gaurav Nigam, ${ }^{2}$ Evan Kontopantelis, ${ }^{3,4} \mathrm{Bu}$ Hayee,

${ }^{1,2}$ Jimmy Limdi. 'Pennine Acute Hospitals NHS Trust, Manchester, UK; ${ }^{2}$ University of Manchester, Manchester, UK; ${ }^{3}$ Kings College Hospital, London, UK; ${ }^{4}$ Kings College London, London, UK

\subsection{6/gutjnl-2019-BSGAbstracts. 166}

Introduction A growing body of evidence supports use of therapeutic drug monitoring (TDM) in improving efficacy and cost-effectiveness of anti-TNF therapy in patients with inflammatory bowel disease (IBD), supported by AGA guidelines. ${ }^{1}$ Existing and evolving knowledge of TDM in clinical practice is less well understood. Our objective was to assess attitudes and barriers to TDM use with anti-TNF's in the UK.

Methods A 17-question survey was distributed to members of the British Society of Gastroenterology. Information on clinician characteristics, demographics, use and barriers towards 
implementing TDM with anti-TNF's was collected. Logistic regression was used to predict factors influencing TDM use.

Results 243 respondents participated $(51.6 \%$ male) of which 237 respondents met inclusion criteria; treating $>5$ IBD patients and at least 1 with an anti-TNF per month. Of the total respondents, $45 \%$ were Consultant Gastroenterologists (GI), 40\% IBD Nurse Specialists (CNS) and 15\% GI Specialist Registrars (SPR). Of these 237 respondents, TDM was used by $95.7 \%$ for secondary loss of response; $71.4 \%$ for primary non-response and $53.6 \%$ used TDM proactively. Barriers for TDM use were time lag in receiving results $(27.1 \%)$, lack of awareness of guidelines (15.6\%), and cost (11.9\%). Clinicians working at a teaching hospital were more likely to use TDM compared to a district hospital (OR 2.6, 95\% CI 0.71-9.8). IBD CNS and GI SPR used TDM more often, when compared to Consultant GI (OR 2.6, 95\% CI 0.69-10 \& OR 1.5, 95\% CI $0.3-7.2$ respectively). Clinicians practising for $>20$ years were more likely to check TDM than less experienced clinicians (OR 4.1, 95\% CI 0.4-41.8). Clinicians with large volume IBD practice ( $>50 \%$ IBD patients per month) were more likely to check TDM than those seeing fewer IBD patients (OR 45.6, 95\% CI 7.5-275). Proactive TDM was more likely to be used by clinicians working in a tertiary care setting (OR 2.25, 95\% CI 0.84-6.05), IBD CNS (OR 1.2, $95 \%$ CI $0.6-2.1$ ), clinicians managing large volume IBD practice (OR 10.8, 95\% CI 1.2-90) and clinicians with 5-9 years of experience in practice (OR 2.6 \& CI 1.04-6.42).

Conclusions Large volume IBD centres with more experience of treating IBD patients are more likely to employ treatmentoptimising strategies with TDM. Significant barriers to TDM implementation in the UK are time lag from test to result, lack of awareness of current guidelines and evolving knowledge, cost and less experience. Validation of point of care testing, lower cost assays, and wider dissemination of current evolving paradigms with updated recommendations may further optimise treatment with anti-TNF therapies.

\section{REFERENCE}

1. Feuerstein JD, et al. Gastroenterology 2017;153(3):827-834.

\section{PTH-108 THE HIDDEN BURDEN OF FAECAL INCONTINENCE IN ACTIVE AND QUIESCENT ULCERATIVE COLITIS: AN UNDERESTIMATED PROBLEM?}

${ }^{1}$ Gaurav B Nigam*, ${ }^{1}$ Jimmy K Limdi, ${ }^{2}$ Shaheen Hamdy, ${ }^{3}$ Dipesh H Vasant. ${ }^{1}$ Pennine Acute Hospitals; ${ }^{2}$ Salford Royal Foundation Trust; ${ }^{3}$ Manchester University Foundation Trust

\subsection{6/gutjnl-2019-BSGAbstracts.167}

Introduction Despite advances in Ulcerative Colitis (UC) therapies, many patients suffer refractory defaecatory symptoms in the absence of active inflammation. For this group, treatment remains challenging, with a paucity of research and limited therapeutic options. In this prospective, ongoing study, we aim to determine the prevalence of faecal incontinence (FI) in patients with quiescent UC.

Methods In a cross-sectional study, consecutive patients with UC attending Inflammatory Bowel Disease (IBD) clinics were invited to participate. Patients completed a series of validated questionnaires; including an IBD-specific FI questionnaire (ICIQ-IBD questionnaire), Hospital Anxiety and Depression
Scale (HADS), the Rome IV diagnostic questionnaire, and the IBD-control questionnaire. Participants were requested to return a Faecal Calprotectin (FCP) within 2 weeks of completing questionnaires. Quiescent UC was defined as IBD-control 8 score $\geq 13$ and IBD-control-VAS $\geq 85$, and/or FCP levels $\leq 250$ (where available, FCP data were used in preference to IBD-control to classify UC activity). Data were compared between active and quiescent groups using chi-square and non-parametric tests.

Results Overall, $n=97$ UC patients $(n=50$ males, mean age 48 (range 18-82) participated. ICIQ-IBD data revealed that most patients experience FI (84/97 (87\%) during 'relapses'. Interestingly, 58/97 (60\%) reported FI when in 'remission', and this group had higher median HADS depression $(\mathrm{P}=0.0002)$, poorer QoL scores $(\mathrm{P}<0.0001)$, and trend towards higher HADS anxiety $(\mathrm{P}=0.09)$ scores, compared to those without FI. Disease activity data (IBD-control and/or FCP) were available for all patients, and based on these 61/97 (63\%) had quiescent UC. The prevalence of FI based on ICIQ-IBD did not differ between those with active $(22 / 36,61 \%)$ and quiescent UC $(36 / 61,59 \%), P=N S$. In those with FI on ICIQ-IBD, median IBD-FI symptom scores, IBD-FI QoL scores and HADS (anxiety: $\mathrm{P}=0.47$, depression: $\mathrm{P}=0.18$ ) did not differ between disease activity groups. However, within the quiescent group, patients that met the more stringent Rome IV criteria for FI $(n=13)$ had higher median IBD-FI symptom scores $(\mathrm{P}=0.007)$ and HADS-depression scores $(\mathrm{P}=0.05)$, a trend to worse IBD-FI QoL $(\mathrm{P}=0.07)$, but similar HADS-anxiety $(\mathrm{P}=0.68)$.

Conclusion This study is one of the first to identify that regardless of disease activity, FI affects most patients with UC, detrimentally impacting patients' psychological wellbeing, impairing their QoL, and should therefore routinely be screened for in clinics. There is an urgent need for further research in the often neglected area of FI and quiescent disease.

\section{PTH-109 FATIGUE IN PATIENTS WITH CROHN'S DISEASE ON LONG TERM VITAMIN B12}

${ }^{1}$ Rupali Patel ${ }^{*}$, 'Eleanor Whittingham, ${ }^{2}$ Lesley Roberts, ${ }^{3}$ sukhdev Singh. ${ }^{1}$ University of Birmingham, birmingham, UK; ${ }^{2}$ University of Warwick, Warwick, UK; ${ }^{3}$ Good Hope Hospital, Birmingham, UK

\subsection{6/gutjnl-2019-BSGAbstracts. 168}

Introduction Some individuals with Crohn's Disease who take long term three monthly Vitamin B12 injections complain of lack of energy and tiredness prior to their next B12 injection. Some have persuaded their General Practitioners to give the B12 injection more frequently than 3 monthly.

Methods We wanted to determine if this was a common experience,and also if seen in other causes of B12 deficiency besides Crohn's Disease.

453 patients on long term B12 in the community were invited to complete a questionnaire, 148 agreed. Most of these patients were elderly with a history of pernicious anaemia. 445 patients completed the questionnaire online. Most of these patients had Crohn's disease. 593 questionnaires were analysed. 
Results In the period prior to Vitamin B12 injection, the majority reported tiredness, lack of energy, poor concentration and mood. Selected control symptoms were only seen in $1 / 4$, figure 1. Of those noticing fatigue, $65 \%$ noticed this in the week prior to B12 injection. The community recruited group and the online group showed a similar pattern of complaints, but the incidence was lower in the Community group.

Following B12 injection there was an improvement in the majority, in energy, concentration, fatigue and mood.

Multivariate analysis to identify factors differing between high and low symptom sufferer, showed only age and treatment interval which predicted greater symptom burden but this only explained a very small proportion of the variation seen (less than 9\%)

A small proportion reported worsening of symptoms following B12 injection; the most common symptom was feeling cold in $7 \%$.

Conclusions In summary, fatigue, lack of energy, poor mood, and difficulty in concentration are seen frequently as individuals approach the 3 monthly B12 injection both in Crohn's Disease and pernicious anemia. These improve following injection

\section{PTH-110 COLITIS ON CT - DOES THIS MEAN INFLAMMATORY BOWEL DISEASE?}

Rajan Patel, Shashank Ramakrishnan, Ella- Portia Veglio-Taylor, Zohaib Tariq, Jonathan King, Kalpesh Besherdas. Royal Free London NHS Trust, London, UK

\subsection{6/gutjnl-2019-BSGAbstracts. 169}

Introduction Cross sectional imaging is commonly used to assess the abdomen for a variety of symptoms. Colitis reported on CT has become a frequent indication for lower gastrointestinal endoscopy. The outcomes of performing colonoscopy for radiology reported colitis is not clearly known.

Methods A retrospective, single centre study of patients referred for a colonoscopy with the indication of 'abnormal imaging'. Data was collected using the endoscopy software audit tool over a 12-month period (September 2017 to August 2018). Patients who had undergone an imaging modality other than CT and those with an overt colonic polyp or mass on CT were excluded from the analysis. Analyses were performed using chi-square and student t-test.

Results 249 patients (183 CT (73.5\%), 66 CTVC (26.5\%)) underwent a colonoscopy for CT evidence of mural thickening (218 (87.6\%)), fat stranding (88 (35.3\%)), inflammation (104 $(41.8 \%))$ or local lymph nodes (37 (14.9\%)); median age 68 (IQR 53 - 79); median time from CT to endoscopy 33 days (IQR 12.5 - 56.5). Initial indication for CT examination: Abdominal pain 112 (45.0\%), Change in bowel habit 39 (15.7\%), ?Malignancy 32 (12.9\%), PR bleeding 13 (5.2\%), Weight loss 9 (3.6\%) and Other 44 (17.7\%).

$53(21.3 \%)$ patients had completely normal lower GI endoscopy. 111 (44.6\%) had uncomplicated diverticulosis, 11 (4.4\%) diverticulitis, 20 (8.0\%) haemorrhoids and 37 (14.9\%) colorectal polyps.

20 patients $(8.0 \%)$ had endoscopic evidence of colitis; 14 (6\%) histological evidence of colitis. 10 (4\%) confirmed IBD at 6 months follow up (4 UC, $6 \mathrm{CD}$ ).

Abstract PTH 110 Table 1 Comparison of endoscopic diagnoses according to CT features and blood results

\begin{tabular}{lllll}
\hline & $\begin{array}{l}\text { Normal } \\
(\mathbf{n}=53)\end{array}$ & $\begin{array}{l}\text { Colitis } \\
(\mathbf{n}=20)\end{array}$ & $\begin{array}{l}\text { Malignancy } \\
(\mathbf{n}=\mathbf{2 1})\end{array}$ & * $\mathrm{p}$ value \\
\hline Age, mean & 63.6 & 54.4 & 69.5 & $<0.02$ \\
$\begin{array}{l}\text { Time to endoscopy (days), } \\
\text { mean }\end{array}$ & 45.3 & 24.5 & 24.9 & $<0.04$ \\
Mural thickening (\%) & $48(90.1)$ & $19(95)$ & $20(95.2)$ & $\mathrm{ns}$ \\
Fat stranding (\%) & $14(26.4)$ & $12(60)$ & $9(42.9)$ & $<0.03$ \\
Inflammation (\%) & $16(30.2)$ & $12(60)$ & $9(42.9)$ & 0.06 \\
Lymph nodes (\%) & $4(5.7)$ & $8(40)$ & $11(52.4)$ & $<0.00006$ \\
Haemoglobin (g/L), mean & 128.0 & 123.8 & 112.2 & 0.005 \\
CRP (mg/L), mean & 29.8 & 55.5 & 68.1 & 0.10, \\
& & & & $<0.05$ \\
\hline
\end{tabular}

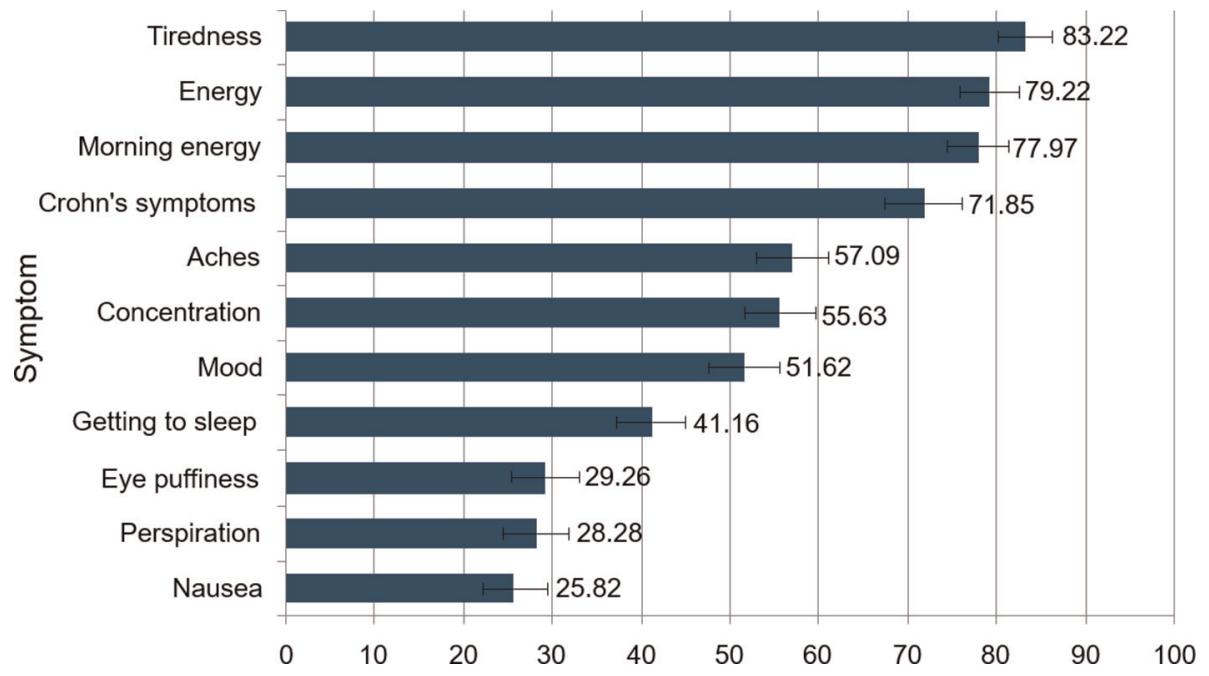

Abstract PTH-109 Figure 1 
Conclusions Colitis reported on CT correlates with endoscopic colitis in only $8 \%$ of patients in this study. Less than $5 \%$ are diagnosed with IBD at 6 months follow up. The correlation improves in younger patients and with shorter interval between CT and endoscopy. One in five patients had completely normal endoscopy and over $90 \%$ had a benign diagnosis. Radiological reporting of fat stranding was an independent risk factor for endoscopic colitis. Anaemia and raised CRP helps identify those at higher risk of malignancy whilst raised CRP alone shows a trend towards identifying true colitis. We conclude that the findings of 'colitis' on CT does not imply IBD in the majority.

\section{PTH-111 IBD NURSE LED RAPID REFERRAL PATHWAY FOR SUSPECTED IBD - IS IT EFFECTIVE?}

1,2Jessica Posso*, 'Vanessa Cambridge, 'David Walker. 'Royal United Hospitals Bath NHS Foundation Trust, Bath, UK; ${ }^{2}$ University of Bristol, Bristol, UK

\subsection{6/gutjnl-2019-BSGAbstracts. 170}

Introduction Recommendations from the UK IBD Standards Group stress the importance of adequate referral pathways in maintaining a high quality of clinical care. In 2016 the gastroenterology department at the Royal United Hospital Bath developed a new rapid referral pathway for suspected inflammatory bowel disease (IBD) patients in order to improve patient care. All GP referrals for suspected IBD were evaluated by a gastroenterology consultant. The clinical information together with any available blood and stool results were reviewed. Symptomatic patients with a suspected new diagnosis of colitis were triaged to an urgent IBD nurse appointment (within 4 weeks) and if appropriate, an urgent flexible sigmoidoscopy with the IBD nurse was offered later the same day. Those patients with a new diagnosis of IBD were given treatment and educational information post procedure. The primary aim of the study was to assess the effectiveness of the new rapid referral pathway and to see if it could be improved further.

Methods A retrospective review was performed of all suspected IBD referrals that had been triaged to an urgent IBD nurse appointment over a 1 year period. For each case identified, a structured proforma review was performed using the original GP referral, the patient's medical notes, blood profile, stool analysis, endoscopy report and histology.

Results 30 patients were referred via the rapid access pathway over a 1 year period. All 30 patients had a flexible sigmoidoscopy with biopsies performed. $30 \%(9 / 30)$ had a new diagnosis of IBD [8 UC, 1 IBD-U], 3\% (1/30) had diverticulitis and $67 \%(20 / 30)$ had a normal procedure. In $93 \%$ of cases bloods including a FBC \& CRP had been performed at the time of referral. However stool microscopy was only performed in $60 \%$ of cases and a faecal calprotectin (FC) in $37 \% .83 \%$ of patients were seen within 4 weeks and all patients were seen within 6 weeks.

Conclusions At the Royal United Hospital Bath, there has been a significant year on year increase in the number of 2WW referrals which has negatively impacted on the ability to see suspected IBD patients within the recommended 4 week period. The new IBD nurse led rapid access pathway resulted in $83 \%$ of suspected IBD patients being reviewed within 4 weeks and all suspected IBD patients being reviewed within 6 weeks. Interestingly, only $30 \%$ of patients were found to have a new diagnosis of IBD. The study showed that FC testing was only happening in a minority of cases. The importance of FC testing has since been highlighted at educational meetings with local GPs and a more rigid IBD referral proforma has been developed. This study shows that an IBD nurse led rapid referral pathway can improve the patient experience by facilitating a quicker diagnosis of IBD and enhances continuity of care.

\section{PTH-112 EFFECT OF CO-MORBIDITIES IN CROHN'S DISEASE ASSOCIATED URINARY METABOLIC PROFILES}

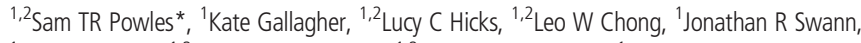
${ }^{1}$ Elaine Holmes, ${ }^{1,2}$ Horace RT Williams, ${ }^{1,2}$ Timothy R Orchard. ${ }^{1}$ Imperial College London, London, UK; ${ }^{2}$ Imperial College Healthcare NHS Trust, London, UK

\subsection{6/gutjnl-2019-BSGAbstracts.17}

Introduction Distinct metabolic signatures have been detected in urine that differentiate Crohn's disease (CD) from controls in multiple studies, with consistent discriminatory metabolites derived from bacteria and co-bacterial pathways (Williams, 2009. AJG). Multiple other diseases have also been found to affect the urinary metabolome, and many of these relate to changes in bacterial associated metabolites (Lu, 2013. Front. Med.). This study aimed to examine a real life cohort of CD patients, and so included patients with other co-morbidities, to examine if the same metabolite changes were present, and if these patients could be distinguished from controls despite the presence of co-morbidities.

Methods Nuclear magnetic resonance ( $\left.\mathrm{H}^{1} \mathrm{NMR}\right)$ spectroscopy was used to acquire urinary metabolic data from $74 \mathrm{CD}$ patients and 100 controls. 19 of the CD group and 48 of the controls had at least one significant co-morbidity (diabetes, asthma, hypertension). Multivariate analysis was performed using OPLSDA. Univariate analysis was also performed to assess whether bacterial associated metabolites, as demonstrated in previous studies (Williams, 2009), were significantly different in CD patients compared to controls. These metabolites were Hippurate, Alanine, Citrate, P-Cresol, Phenyacetylglutamine (PAGn), and Dimethylglycine (DMG). 